\title{
Conducta financiera del venezolano: Una escala para contextos de adversidad económica
}

\section{Venezuelan Financial Behavior: A Scale for Economic Adversity Contexts}

\author{
Antonio Martins * 1, Luis Rodríguez ${ }^{2}$ \\ 1 - Escuela de Psicología, Universidad Católica Andrés Bello, Caracas, Venezuela. \\ 2 - Centro de Investigación y Evaluación Institucional, Universidad Católica Andrés Bello, Caracas, Venezuela.
}

Introducción

Metodología

Resultados

Discusión

Referencias

Recibido: 18/09/2021 Revisado: 27/11/2021 Aceptado: 08/12/2021

\section{Resumen}

La conducta financiera es entendida como cualquier acción que implique el uso del dinero. Estos comportamientos pueden variar en función de la realidad económica de los países, siendo los territorios con dificultades financieras los que imponen mayores retos en su medición. Se planteó como objetivo desarrollar una escala de conducta financiera y analizar sus propiedades psicométricas. Los ítems fueron elaborados con la colaboración de jueces expertos, y las dimensiones de la conducta financiera fueron: (a) conducta de ahorro, (b) manejo de deuda y (c) manejo del dinero. La muestra estuvo conformada por 780 participantes $(59.74 \%$ femeninos) caraqueños con edades comprendidas entre los 18 y 70 años, provenientes de distintos sectores ocupacionales. Se obtuvo una adecuada consistencia interna del instrumento en la mayoría de los factores. Además, se halló evidencia de validez externa al test. Se muestran en el estudio diferencias en las tres dimensiones en función de variables sociodemográficas.

Palabras clave: conducta financiera, teoría clásica de los test, ahorro, manejo de la deuda, psicología económica

\begin{abstract}
Financial conduct is understood as any action that involves the use of money. These behaviors may vary depending on the economic reality of the countries, being the territories with financial difficulties those that pose greatest challenges in their measurement. The objective was to develop a financial behavior scale and analyze its psychometric properties. The items were prepared with the collaboration of expert judges and the dimensions of financial behavior were: (a) savings behavior, (b) debt management and (c) money management. The sample consisted of 780 participants $(59.74 \%$ female) from Caracas aged between 18 and 70 years, from different occupational sectors. An adequate internal consistency of the instrument was obtained in most of the factors. In addition, evidence of external validity to the test was found. Differences in the three dimensions depending on sociodemographic variables are shown in the study.
\end{abstract}

Keywords: financial behaviour, classical test theory, savings, debt management, economic psychology 


\section{Introducción}

En la actualidad existe un gran interés por conocer con mayor detalle cómo las personas piensan y se comportan respecto a sus recursos económicos. Las explicaciones de dichos comportamientos se remontan al desarrollo teórico de la economía en el siglo XVIII; Adam Smith, el padre de la economía clásica, ya realizaba para aquel entonces análisis económicos en los que se consideraban conceptos conductuales (Laibson \& List, 2015).

Sin embargo, la aproximación de los economistas se basó en la noción del homo economicus, que proponía que los seres humanos han de comportarse económicamente de una manera racional. No obstante, con el paso del tiempo y los avances en la investigación, estos postulados fueron perdiendo popularidad progresivamente. En parte, los estudios del psicólogo Daniel Kahneman (2013) contribuyeron a un cambio de paradigma, a partir del cual se formó la denominada economía conductual y la psicología económica (Kirchler \& Hoelzl, 2018).

Esta última es entendida como una rama de la psicología aplicada que busca estudiar la vida mental económica y el comportamiento de los sujetos, teniendo a su vez una estrecha vinculación con la economía, por lo que se puede considerar como un campo interdisciplinar en el que se busca explicar cómo se dan las tomas de decisiones económicas; entre estas, el ahorro, gasto, toma de riesgos financieros, así como sus diversas causas y consecuencias (Ranyard \& De Mello-Ferrerira, 2017). Es dentro de esta área que se enmarca la conducta financiera, el constructo principal de la presente investigación.
Definición y teorías de la conducta financiera

Para definir el concepto de conducta financiera, primero es conveniente señalar que no hay un consenso en las teorías, motivo por el cual es necesario precisar: ¿qué es una conducta? La conducta se entiende como la actividad física de un organismo ante estímulos externos o internos; que incluye actividades objetivamente observables, actividades introspectivas observables y procesos no conscientes (VandenBos, 2015). De aquí es importante resaltar que es una acción con algún parámetro observable.

Partiendo de este marco interpretativo, Xiao (2008) define la conducta financiera como cualquier acción humana que es relevante en el manejo de dinero, siendo sus dimensiones aquellas referidas a la administración de efectivo, del crédito (deuda) y del ahorro. También abarca la adquisición de seguros, la planificación de retiro, la inversión y el pago/planificación de impuestos (Van Raaij, 2016).

Existen múltiples teorías en el área de la psicología económica desarrolladas con el fin de explicar la conducta financiera. A continuación, se presentan algunas de estas propuestas, sin pretensión de exponer todas las teorías existentes ni ser extensivo en su tratamiento.

\section{Modelo de la socialización financiera familiar:} sostiene que el proceso de interacción con el entorno social es fundamental para el desarrollo de conductas financieras efectivas. Las relaciones entre las características del grupo familiar, los factores sociodemográficos (edad, ingreso y sexo) y los antecedentes personales generan los resultados de la socialización financiera. Estos resultados son actitudes, conocimientos y capacidades económicas que influyen sobre la conducta financiera, y estas, a su vez, sobre el bienestar monetario (Gudmunson \& Danes, 2011). 
Es importante aclarar sobre este modelo que, si bien dichos procesos inician en la infancia, en la que se adquieren repertorios y se desarrollan patrones de comportamiento financiero, es durante la adultez cuando podría ocurrir una modificación en dichos patrones, en especial en periodos o eventos de grandes cambios (Gudmunson, Ray, \& Xiao, 2016). Esto denota que la conducta financiera es de carácter dinámico, ya que puede modificarse en función del contexto económico.

Modelo de Van Raaij: es una propuesta caracterizada por las relaciones entre las variables psicológicas y económicas. Presenta cuatro factores principales que de forma cíclica dan cuenta de la dinámica de la conducta financiera: el ambiente económico (E) influye en el ambiente percibido (E/P), y este, a su vez, sobre la conducta económica (B), que impactará en el bienestar subjetivo (SW), el cual termina afectando al ambiente económico. Adicionalmente hay variables externas que interactúan de forma individual con cada una de estas, siendo correspondientemente el contexto general en $\mathrm{E}$, el factor personal en $\mathrm{E} / \mathrm{P}$ (edad, sexo e ingreso), el factor situacional en $\mathrm{B}$ y el descontento social en SW (Van Raaij, 1981).

De esta manera, la conducta no es solo una consecuencia (del E/P) sino que puede ser también una causa (del SW y del E). Los modelos de socialización financiera y el de Van Raaij permiten dar cuenta de la explicación de la conducta, pero no de su composición; por lo tanto, se consideran como dimensiones las expuestas por Xiao (2008) y Van Raaij (2016).

\section{Formas de medición de la conducta financiera}

Se ha advertido que al momento de trabajar con este tipo de conducta se deben precisar ciertos puntos: (a) decidir si trabajar con la conducta propiamente o con los productos de la conducta; (b) decidir si trabajar con una conducta particular o generar una categoría de agrupación; (c) definir los elementos de la conducta, tales como la acción, el objetivo, el contexto y el tiempo de duración; (d) decidir si medir mediante autoinforme u observación, y, por último, (e) establecer la medida de conducta, por ejemplo, de forma binaria, categoría o frecuencia. Sobre esta última se aclara que debe ser elegida en función de las preguntas (Xiao, 2008).

Sobre el autoinforme se debe ser cuidadoso, ya que problemas como la deseabilidad social o aquiescencia de respuesta generan que los sujetos distorsionen sus planteamientos, llegando a mentir (Magnusson, 1972). Sin embargo, a veces es la única opción realizable, dado que hay conductas que no se pueden observar directamente.

Consecuentemente con tales planteamientos, se ha advertido que existen formas de disminuir los riesgos que suponen los autoinformes, Mcnair y Crozier (2017) recomiendan invertir algunos ítems o mantener estandarizada la situación de evaluación para evitar este tipo de inconvenientes.

\section{Trabajos previos de medición del constructo}

La mayoría de las investigaciones, cuando trabajan con conducta financiera como variable, no cuentan con escalas psicométricamente adecuadas, ya que suelen ser mediciones ad hoc o instrumentos que no informan indicadores psicométricos. Por ende, en la presente investigación se seleccionaron como antecedentes aquellas escalas de conducta financiera que sí cuentan con un mínimo de datos psicométricos (ver Tabla 1). 
Tabla 1

Comparación de las escalas de conducta financiera.

\begin{tabular}{|c|c|c|c|c|c|}
\hline Escala & Factores & Confiabilidad & \# de ítems & $\mathbf{N}$ & Autores y año \\
\hline \multirow{4}{*}{$\begin{array}{l}\text { Escala de Conducta de } \\
\text { Gestión Financiera (ECCF) }\end{array}$} & Ahorro e inversión & $\alpha=.78$ & 5 & \multirow{4}{*}{1011} & \multirow{4}{*}{$\begin{array}{l}\text { Dew y Xiao } \\
(2011)\end{array}$} \\
\hline & Adquisición de seguro & $\alpha=.73$ & 3 & & \\
\hline & Manejo de dinero & $\alpha=.63$ & 4 & & \\
\hline & Crédito & $\alpha=.57$ & 3 & & \\
\hline $\begin{array}{l}\text { Habilidades de Gestión del } \\
\text { Dinero (EHGD) }\end{array}$ & General (ad hoc) & $\alpha=.86$ & 9 & 271 & $\begin{array}{l}\text { Garðarsdóttir y } \\
\text { Dittmar (2012) }\end{array}$ \\
\hline \multirow{4}{*}{$\begin{array}{l}\text { Escala Breve de Gestión del } \\
\text { Dinero (EBGD) }\end{array}$} & Ahorro & $\alpha=.89$ & 4 & \multirow{4}{*}{1078} & \multirow{4}{*}{$\begin{array}{l}\text { Ksendzova, } \\
\text { Donnelly y } \\
\text { Howell (2017) }\end{array}$} \\
\hline & Adquisición de seguro & $\alpha=.82$ & 5 & & \\
\hline & Crédito & $\alpha=.80$ & 5 & & \\
\hline & Manejo de dinero & $\alpha=.79$ & 4 & & \\
\hline
\end{tabular}

Cabe destacar que la EHGD trabaja las conductas de gestión a nivel general y, si bien informa una confiabilidad elevada, no cuenta con indicadores de validez de contenido y criterio. En otro orden de ideas, Porter y Garman (1993) plantean un conjunto de ítems donde aparte de las dimensiones de manejo de dinero, crédito y riesgo (adquisición de seguro), dividen la dimensión ahorro en acumulación de capitales (ahorro tradicional) y jubilación/planificación de retiro. También establecen una dimensión de gestión general que incluye conductas de planificación e impulsividad. Esta propuesta, aunque es una categorización que podría ser relevante a nivel heurístico, no cuenta con indicadores psicométricos que den cuenta de la utilidad del instrumento en distintas muestras.

Esto último invita a que el análisis se centre en la ECCF y la EBGD, ya que son muy similares. Respecto al manejo de crédito, se encuentra que en la segunda escala la confiabilidad para esta dimensión aumenta, lo que se puede deber no solo al aumento del número de ítems (Magnusson, 1972) sino también a la inclusión de conductas generales como endeudamiento o mayor cantidad de egresos que de ingresos, los cuales no están supeditados necesariamente a las tarjetas de crédito, aspecto que sí ocurre en la ECCF.

En cuanto a la dimensión ahorro, ambas escalas tienen una confiabilidad similar. Igualmente, el porcentaje de varianza de esta dimensión es el mayor. Por otra parte, respecto a la dimensión manejo de dinero, es la que menor confiabilidad y porcentaje de varianza explica. Sin embargo, la EBGD tiene una confiabilidad mucho mayor que la ECCF. En otro orden de ideas, la ECCF cuenta con validez convergente adecuada al correlacionarla con los ítems de Perry y Morris (2005).

Respecto a su aplicación en otros países, la escala de ECCF fue validada en Brasil, tomando como muestra 195 estudiantes graduados y profesores de postgrado. En el análisis factorial confirmatorio se obtuvieron los cuatro factores de la escala original; asimismo el instrumento tomado de forma unidimensional resultó confiable (confiabilidad compuesta $=.84$ ). Respecto a la validez, la escala correlacionó negativamente con tensión 
financiera y con compra impulsiva (Veiga, Avelar, Moura, \& Higuchi, 2019).

Por último, en cuanto a las variables que se han relacionado en la literatura con la conducta financiera, se encuentra que la impulsividad de la compra correlaciona negativamente con ella (Dew \& Xiao, 2011; Veiga et al., 2019), mientras que los hábitos de compra se asocian de forma positiva con la conducta positiva, dado que es un manejo responsable y cuidadoso del dinero (Van Raaij, 2016).

\section{Crisis económica y conducta financiera}

Una vez repasados brevemente los principios de la conducta financiera, se debe aclarar un punto importante sobre esta: los comportamientos financieros de las personas naturalmente se ven afectados por el contexto en el que se desenvuelven los individuos (Gärling, Kirchler, Lewis, \& Van Raaij, 2009; Van Raaij, 1981). Es por ello que resulta necesario visualizar el contexto económico del venezolano durante las dos primeras décadas del siglo XXI. Lo primero a mencionar es que revisiones exhaustivas de la historia del país suelen concluir que la economía venezolana posee un carácter impredecible (Sardi, Angelucci, Martins, \& Peña, 2020).

Para comprender las condiciones económicas actuales, resultan ilustrativos los hallazgos de la Encuesta Nacional de Condiciones de Vida (ENCOVI), que han reflejado longitudinalmente un empobrecimiento masivo de los venezolanos: mientras que para el año 2015 un 87\% de los hogares se hallaba por debajo de la línea de la pobreza, para el año 2019 se estimó que este porcentaje aumentó a 96.2\% de la población (UCAB, 2020).

Ante esta realidad, el gobierno nacional ha planteado una serie de políticas económicas que han redundado en el moldeamiento de las con- ductas financieras que pueden adoptar los venezolanos. Lo más llamativo es que a partir del año 2018, se ha producido un viraje en cuanto al manejo de las divisas en el país. En este año el gobierno facilitó la libre convertibilidad de la moneda extranjera en toda Venezuela, involucrando en estas transacciones al Sistema Bancario Nacional (Olmo, 2019).

Relacionadas con las operaciones cambiarias y el proceso desarrollado por el gobierno para controlar el dólar paralelo, se implementaron una serie de políticas bancarias referidas al encaje legal. Solo desde el año 2018 al 2019 el encaje pasó de ser $21.5 \%$ a $100 \%$ (Gaceta Oficial 4443.040); esto se tradujo en que los bancos no pudieron otorgar créditos a empresas ni a particulares con el fin de no emitir mayor cantidad de bolívares sin sustento, así como para controlar la inflación (Barbar, Benasayag, \& Salomón, 2019). Esto limitó el acceso y uso de tarjetas de crédito en la banca nacional.

Ambas políticas referidas al manejo de las divisas y a la asignación de créditos han implicado cambios en la manera en la que ahorran los venezolanos y en la forma en la que se aproximan a sus deudas mediante los préstamos formales e informales. De manera concreta, aunque la posibilidad de ahorro se ve reducida en esta población empobrecida, cuando esta aparece, se da a través de la acumulación de divisas (y no de bolívares, moneda de curso oficial que ha visto pulverizado su valor frente al dólar) o la obtención de bienes duraderos; mientras que la posibilidad de obtención de créditos se ha visto reducida a aquel pequeño porcentaje de la población que tiene acceso a la banca internacional.

Ante este contexto, resulta necesaria la elaboración de una escala que, aunque parta de las concepciones tradicionales en la medición de la conducta financiera (Dew \& Xiao, 2011; Ksendzova et al., 2017) considere las particula- 
ridades de las economías inestables tales como el uso de divisas para ahorrar, solicitud de deudas no convencionales (préstamos de familiares o amigos) y consumo impulsivo en moneda local; y proporcione de esta manera una propuesta operacional que resulte aplicable para situaciones que tengan como denominador común la adversidad económica, la cual no es abordada por las escalas tradicionales expuestas anteriormente.

En la literatura se ha encontrado que, en economías inestables, donde predominan la inflación y la recesión, las conductas financieras que adoptan los individuos son disímiles a las adoptadas por sujetos inmersos en economías estables. Por ejemplo, para el caso del ahorro, lo recomendable es que ante tasas inflacionarias elevadas las personas eviten ahorrar, ya que cualquier acumulación de dinero es superada por el aumento de los precios (Van Raaij, 2016). Por tanto, lo favorable resultaría ser el gasto rápido de la moneda local o el ahorro en bienes duraderos o en una moneda con mayor estabilidad.

Con respecto a la deuda, propuestas operacionales como las de Dew y Xiao (2011) consideran que pagar la totalidad de las deudas antes de su vencimiento resulta adaptativo. No obstante, en contextos inflacionarios, lo más eficiente sería acumular la mayor cantidad de deuda posible en la moneda devaluada y retrasar el pago en el tiempo, dado que la tasa de inflación será superior al interés de la deuda.

Por último, en cuanto al manejo del dinero en este tipo de situaciones económicas excepcionales, las personas tienden a realizar un ajuste en su patrón de consumo con el objetivo de mantener el valor de sus ingresos. Por ejemplo, los sujetos ante la adversidad buscan comprar los productos más baratos, de mayor calidad o venden bienes duraderos (Gärling et al., 2009).

Partiendo de estos hallazgos atípicos, que indican que situaciones culturales o contextuales requieren de una forma de medición del constructo que sea diferente a las que se tienen, se ha considerado necesario construir un test (Fernández, Pérez, Alderete, Richaud, \& Liporace, 2010). Dicha construcción está enmarcada en los postulados de la teoría clásica de los test (De Minzi, 2008; Magnusson, 1972), por lo que en la presente investigación se plantean los siguientes objetivos:

1) Construir un instrumento con adecuada validez de contenido para la medición de la conducta financiera en contextos de adversidad económica.

2) Evaluar la estructura factorial del test mediante la realización de un análisis factorial exploratorio, que dilucide si existe una medición de la conducta financiera en términos de las dimensiones constituyentes, útiles para contextos adversos: (a) ahorro, (b) manejo de la deuda y (c) manejo del dinero.

3) Obtener evidencia de confiabilidad mediante el método alfa de Cronbach que evalúa la consistencia interna de las puntuaciones del test.

4) Obtener evidencia de validez externa del test a través de procedimientos de convergencia y divergencia.

\section{Metodología \\ Tipo y diseño de investigación}

En cuanto al grado de control que existe sobre las variables sometidas a estudio, la investigación fue de tipo cuantitativa no experimental (Kerlinger \& Lee, 2002), tomando en consideración que no existe una manipulación directa de las variables. Por otro lado, el diseño fue de tipo instrumental (Montero \& León, 2007), ya que la investigación tuvo como objetivo el desarrollo de una escala y la obtención de sus propiedades psicométricas. 


\section{Participantes}

La muestra estuvo conformada por 780 participantes (59.74\% femeninos, 40.26\% masculinos) oriundos de la Gran Caracas y que fueron seleccionados por medio de un muestreo no probabilístico de tipo intencional, ya que no se consideraron técnicas aleatorizadas al momento de elegir a los sujetos. La edad promedio de los encuestados fue de 32 años, con una desviación estándar de 15 años. Con respecto a su ocupación, 28.72\% eran trabajadores del sector privado, $25.77 \%$ estudiantes de universidades privadas, $17.69 \%$ personal de empresas públicas, $14.74 \%$ estudiantes de universidades públicas y un $13.08 \%$ se describió como "patronos, empleadores o trabajadores por cuenta propia". Esta distribución de la muestra permitió conocer que un $72.95 \%$ de la muestra mantenía un trabajo, un $19.23 \%$ recibía remesas y un $44.6 \%$ poseía tarjeta de crédito al momento de la recolección de los datos.

\section{Instrumentos}

Cuestionario de datos sociodemográficos. Este cuestionario fue elaborado con la finalidad de facilitar la identificación de los sujetos en función del sexo, la edad, la posibilidad de mantener un trabajo actual y la ocupación que mantenían al momento de la encuesta. También se indagó si los participantes disponían de préstamos o tarjetas de crédito al momento de la investigación. Fue en este documento que además se solicitó el consentimiento para participar en el estudio, al tiempo que se informó del anonimato de las respuestas. No se incluyó el nivel de ingreso, ya que estudios nacionales (ENCOVI) proponen evitar esta medida dada la resistencia de las personas para declararlos, y, por tanto, se toma la ocupación como un indicador indirecto.
Escala de Conducta Financiera (Martins \& Rodríguez, 2020). Escala creada en contextos de formación académica para un curso básico de psicometría de la Universidad Católica Andrés Bello (UCAB). El instrumento está conformado por 24 reactivos (8 ítems por factor) cuyo formato de respuesta fue tipo Likert de 4 opciones que van desde Nunca a Siempre, con la finalidad de equipar las respuestas dadas ante todos los instrumentos del presente estudio. En la construcción de la tabla de especificaciones, que sirvió de fundamento para el proceso de validación de contenido por medio de la valoración de jueces expertos, se estimó la existencia de tres dimensiones de la conducta financiera: (a) ahorros, (b) manejo de la deuda y (c) manejo del dinero. A mayor puntaje en cada uno de los componentes se estima que mayor será la tendencia al ahorro, la inclinación hacia el uso de créditos formales o informales y la inclinación hacia la planificación racional del dinero respectivamente.

Escala de Hábitos de la Compra y Conductas de Consumo (Denegri, Palavecinos, Ripoll, \& Yáñez, citado en Ortega \& Rodríguez-Vargas, 2004). El instrumento cuenta con 19 reactivos cuyo formato de respuesta es tipo Likert de 4 puntos, que va desde Nunca hasta Siempre. En una muestra de adultos colombianos provenientes de la ciudad de Barranquilla, con un nivel socioeconómico medio y alto, las puntuaciones de la escala presentaron una estructura de tres dimensiones, cuyos coeficientes de consistencia interna resultaron adecuados para fines diagnósticos y de investigación. Estos tres factores extraídos por Ortega y Rodríguez-Vargas (2004) fueron: (a) calidad del producto $(\alpha=.81)$, (b) planificación de la compra $(\alpha=.80)$ y (c) uso responsable del crédito $(\alpha=$ .85). En la muestra de la presente investigación, se obtuvo una consistencia interna adecuada para fines de investigación en la escala total $(\alpha=.72)$. 
Por otro lado, se realizó un análisis factorial exploratorio con mínimos cuadrados no ponderados (ULS robusto) para matrices policóricas con rotación oblimin directa, que resultó en la extracción de dos factores que explicaron el $42.64 \%$ de la varianza total del test y que fueron denominados como: (a) planificación de la compra $(\alpha=$.79) y (b) uso responsable del crédito y calidad del producto $(\alpha=.76)$. A mayor puntaje en cada una de las dimensiones se estima que mayor será la planificación de la compra, el uso responsable del crédito y mejor será la calidad de los productos comprados respectivamente.

Escala de Impulsividad de la Compra (LunaArocas, 1998). La escala se encuentra conformada por 17 ítems con un formato de respuesta tipo Likert de 4 puntos, que va desde Nunca hasta Siempre. En la presente muestra, para las puntuaciones de la escala total se obtuvo un coeficiente de consistencia interna elevado para fines de investigación y diagnóstico $(\alpha=.88)$. Por su parte, en cuanto a la estructura factorial de las puntuaciones del test, un análisis factorial exploratorio ULS robusto para matrices policóricas con rotación oblimin directa, permitió extraer un único factor que explicó un 38.55\% de la varianza total del instrumento. A mayor puntuación en la escala total, mayor será la impulsividad de la compra.

\section{Procedimiento}

Se diseñó una tabla de especificaciones que permitió precisar el contenido o la dimensión que pretendía medir cada uno de los reactivos, $\mathrm{su}$ formato de corrección (directo o inverso) y de respuesta. A partir de ella, los profesores de psicometría de la UCAB, en colaboración con sus respectivos estudiantes, procedieron a construir un banco de ítems que fue sometido al juicio de tres expertos en psicometría, metodología y economía.

El juicio de los expertos se realizó ítem por ítem y se limitó a la consideración de: (a) la pertinencia de los reactivos, (b) su claridad y (c) su adecuación a la muestra. Una vez obtenido el juicio de expertos se procedió a promediar sus decisiones, y aquellos reactivos con baja valoración fueron modificados, siguiendo para ello las sugerencias cualitativas de los jueces.

Una vez obtenida la escala preliminar se procedió a administrar el instrumento para obtener los indicadores psicométricos en la muestra seleccionada. Junto a la escala de conducta financiera, se administró un instrumento de hábitos de compra y otro de impulsividad de la compra para obtener indicadores de validez convergente y divergente. Todas las escalas fueron administradas en formato electrónico a través de la plataforma Google Forms. El proceso de envío de los instrumentos fue realizado por los estudiantes bajo la supervisión de sus profesores y tras ser adiestrados por los investigadores.

\section{Consideraciones éticas}

Previo al proceso de aplicación de los instrumentos, se obtuvo el consentimiento explícito de los interesados para participar en la investigación. El anonimato de los sujetos involucrados, y la garantía de la participación voluntaria y sin coerción fueron otros aspectos éticos considerados, siguiendo las directrices de la Declaración de Helsinki (Manzini, 2000).

\section{Análisis de datos}

Los cálculos estadísticos necesarios para conseguir los objetivos de investigación fueron 
realizados con los softwares estadísticos IBM SPSS 22 (IBM Corp., 2013) y FACTOR Analysis (Lorenzo-Seva \& Ferrando, 2011). Para conocer la estructura factorial subyacente a la escala de conducta financiera, se estimaron varios análisis factoriales exploratorios (AFE) con el método de extracción de mínimos cuadrados no ponderados para matrices policóricas (RULS), con rotación oblimin directa y varimax normalizada. Esto se debió a que era la forma de extracción más adecuada frente a la ausencia de normalidad en algunos ítems - evaluada a partir de la asimetría y la curtosis de los reactivos-, al formato de respuesta Likert de cuatro puntos de la escala y a que evita los casos Heywood (Lloret-Segura, Ferreres-Traver, Hernández-Baeza, \& TomásMarco, 2014). Previamente fueron evaluados y garantizados todos los supuestos requeridos para los AFE. El análisis paralelo, en conjunto con los indicadores de bondad de ajuste, fue empleado como técnica para retener las dimensiones.

En segundo lugar, una vez extraídos los factores subyacentes a las puntuaciones de la escala, se propuso evaluar la consistencia interna de las dimensiones obtenidas a partir del coeficiente alfa de Cronbach. Posteriormente, fueron estimadas correlaciones de Pearson entre las puntuaciones de las dimensiones de la conducta financiera y los indicadores de impulsividad de la compra y hábitos de consumo.

La correlación conducta financiera-impulsividad de la compra fue interpretada como un indicador de validez discriminante, mientras que las asociaciones entre conducta financiera y las dimensiones de los hábitos de la compra sugirieron un proceso de validación convergente, tomando en consideración la inexistencia de escalas previas que evaluaran la conducta financiera tal y como fue propuesta teóricamente en la presente investigación.

Por último, se procedió a realizar un análi- sis exploratorio de datos a partir de las puntuaciones obtenidas en cada una de las dimensiones de la conducta financiera, comparando, el manejo de los ahorros, deuda y dinero en función del sexo, edad y ocupación de los participantes. Técnicas como la $t$ de Student para muestras independientes, correlaciones momento-producto de Pearson y análisis de varianza de una vía (ANOVA) fueron necesarias para llevar a cabo dichas comparaciones.

\section{Resultados \\ Análisis psicométricos}

Para estudiar la estructura factorial del test, en primer lugar, se realizó un análisis paralelo que permitió estimar el número de factores a extraer en el ULS robusto con rotación oblimin directa. La cantidad de factores a extraer fue de tres, esto tomando en consideración que al comparar el porcentaje de varianza en los datos reales con la proporción del percentil 95, se halló que fue hasta la tercera dimensión donde se superó el criterio del azar.

Posteriormente, se procedió a calcular un nuevo AFE bajo los mismos parámetros, pero solicitando la extracción de tres factores con los 24 ítems iniciales. Bajo este cálculo se eliminaron aquellos reactivos cuyas cargas fueron menores a .30. En este sentido, fueron descartados tres ítems (ítem 6: Ahorro gran porcentaje de dinero recibido en bolivares, ítem 8: Pago atrasado a mis amigos o allegados cuando me toca devolver el dinero, ítem 15: Utilizo una proporción de mis ingresos en bolivares para comprar bienes duraderos); este criterio fue utilizado pese a las sugerencias de Lloret-Segura et al. (2014), dado que Hair, Black, Babin y Anderson (2019) indican que en muestras mayores a 350 sujetos pudiera reducirse el criterio de .40 a .30 . 
Con los 21 reactivos restantes, fue calculado un nuevo AFE con rotación oblimin directo. No obstante, tras la verificación de una baja correlación entre los factores extraídos se implementó la estimación de un nuevo análisis con rotación varimax normalizada, siguiendo las pautas de interpretación planteadas por Lloret-Segura et al. (2014): no se encontraron diferencias sustanciales en la distribución y monto de las cargas si se comparan las dos rotaciones.

En este último AFE, al igual que en los anteriores, se garantizó el cumplimiento de los supuestos iniciales. En este sentido, se encontró una alta interrelación entre las variables sometidas al análisis $(\mathrm{KMO}=.84)$, al tiempo que la matriz de correlaciones inicial no se ajustó a la matriz de identidad (test de esfericidad de Bartlett = 7017.70; $p<0.001)$. Por otra parte, los índices de bondad de ajuste demostraron niveles adecuados $(\mathrm{RMSEA}=.045 ; \mathrm{GFI}=.981 ; \mathrm{CFI}=.978)$.

Estos tres factores extraídos explicaban el $49.69 \%$ de la varianza total del test, y en la Tabla 2 se muestra la configuración de cada una de las dimensiones. El Factor 1, denominado ahorro, explicaba el 24.54\%, la segunda dimensión, llamada manejo de la deuda, representa el $14.61 \%$ de la varianza y, por último, la dimensión de manejo de dinero explicó un $10.54 \%$, y los autovalores para ellos fueron de 5.15, 3.06 y 2.21 respectivamente.

Con respecto a la consistencia interna de las dimensiones del instrumento, fue calculado el coeficiente alfa de Cronbach para los factores extraídos, obteniendo que la correlación ítem-test presentó un recorrido de -.29 hasta .61 , estando la mitad de los reactivos por encima de .30 en su asociación con el test total. Para el caso de la dimensión de ahorro, se obtuvo una consistencia interna adecuada para fines de investigación y diagnóstico $(\alpha=.85)$. En segundo lugar, la dimensión de manejo de la deuda resultó adecuada para fines de investigación $(\alpha=.69)$. Por último, el factor de manejo del dinero demostró una baja consistencia interna $(\alpha=.59)$.

Además, se calculó una matriz de correlaciones (ver Tabla 3) con la finalidad de obtener evidencia de validez convergente y discriminante a partir de la relación de las puntuaciones obtenidas en las tres dimensiones de la conducta financiera con la impulsividad de la compra y los hábitos de esta. El manejo de la deuda y del dinero obtuvieron evidencia de validez discriminante al presentar correlaciones nulas o inversas con la impulsividad de la compra, e indicadores de validez convergente al relacionarse positiva y significativamente con todos los indicadores de los hábitos de consumo.

Por último, las conductas de ahorro se asociaron de forma positiva y baja con la planificación de la compra, siendo esto reflejo de la validez convergente mientras que el indicador de discriminación para dicha dimensión se mostró afectado al obtenerse una correlación positiva y moderada-baja entre el ahorro y la impulsividad de la compra.

\section{Análisis adicionales}

A nivel descriptivo, en las puntuaciones obtenidas por dimensiones de la conducta financiera, se halló que las conductas de manejo del dinero resultaron mayores $(\mathrm{M}=2.93 ; \mathrm{DE}=.59)$ que las presentes en ahorro $(\mathrm{M}=2.83 ; \mathrm{DE}=.71) \mathrm{y}$ manejo de la deuda $(\mathrm{M}=2.51 ; \mathrm{DE}=.68)$. Para las conductas de ahorro, se obtuvo que la compra de monedas extranjeras se constituyó como el medio de ahorro más valorado, seguido por la compra abundante de productos que a posteriori pudieran ver aumentado su valor en bolívares.

Por su parte, para el manejo de la deuda, los participantes tendieron a valorar en mayor medida el pago retrasado de los intereses generados 
Tabla 2

Matriz rotada.

\begin{tabular}{|c|c|c|c|c|}
\hline & Ítems/Factores & 1 & 2 & 3 \\
\hline 1 & $\begin{array}{l}\text { Cuando cuento con dinero en efectivo, llevo mayor cantidad de dólares antes que } \\
\text { bolívares }\end{array}$ & 609 & .072 & -.224 \\
\hline 2 & $\begin{array}{l}\text { Con los bancos nacionales, pago los créditos que dispongo en el tiempo estipulado } \\
\text { (Inverso) }\end{array}$ & .053 & .706 & -.098 \\
\hline 3 & $\begin{array}{l}\text { Compro productos de una manera abundante porque sé que a largo plazo me saldrá más } \\
\text { barato }\end{array}$ & .400 & .199 & .016 \\
\hline 4 & Tengo un límite para el monto de mis transacciones semanales & .008 & .038 & .404 \\
\hline 5 & $\begin{array}{l}\text { Si el interés de mis deudas (con bancos o personas particulares) es en bolívares, pago } \\
\text { en las fechas límites }\end{array}$ & .024 & .347 & .107 \\
\hline 7 & Administro mi dinero de forma racional, solo gasto en lo necesario & .017 & .104 & .788 \\
\hline 9 & Ahorro en moneda extranjera & .911 & -.056 & -.040 \\
\hline 10 & Junto con mi familia, planificamos el uso del dinero que obtenemos para un futuro & .388 & .126 & .369 \\
\hline 11 & Abono un poco más de lo requerido en los pagos mínimos de mi(s) tarjeta(s) de crédito & -.010 & .681 & .123 \\
\hline 12 & Ahorro dinero en moneda extranjera previniendo emergencias & .932 & -.001 & .071 \\
\hline 13 & Chequeo opciones de precios antes de decidir comprar algún producto & .099 & .106 & .400 \\
\hline 14 & Pido préstamos en bolívares para pagar algunos gastos inmediatos & .036 & .349 & -.111 \\
\hline 16 & Al momento de pagar cosas necesarias, dispongo del dinero para ellas & .337 & .209 & .074 \\
\hline 17 & $\begin{array}{l}\text { Cuando quiero adquirir algo fuera del alcance de mi presupuesto considero los créditos } \\
\text { (formales o informales) como una buena opción }\end{array}$ & .016 & .549 & -.038 \\
\hline 18 & Ahorro parte mi dinero en divisas para distintos fines (ej. educación, viajes, recreación) & .890 & .033 & -.036 \\
\hline 19 & Tengo detallados mis gastos mensuales & .115 & .163 & .527 \\
\hline 20 & $\begin{array}{l}\text { Cuando tengo que pagar algo (con tarjeta de crédito o préstamos) procuro que el interés } \\
\text { sea en bolívares }\end{array}$ & .068 & .687 & .166 \\
\hline 21 & Busco ahorrar lo que pueda en moneda extranjera pensando en el futuro & .911 & .038 & .048 \\
\hline 22 & Compro impulsivamente cosas que no necesito (Inverso) & -.216 & -.030 & .527 \\
\hline 23 & Si aumentaran el límite de mi tarjeta de crédito en bolívares lo gasto inmediatamente & .232 & .521 & -.192 \\
\hline 24 & $\begin{array}{l}\text { Cuando mi grupo familiar dispone de bolívares y no tenemos un gasto pendiente, los } \\
\text { incito a comprar monedas extranjeras }\end{array}$ & .637 & .194 & .066 \\
\hline
\end{tabular}

por los créditos solicitados, siempre y cuando estos fueran adquiridos en bolívares y no en cualquier otra moneda.

Para el caso de la edad, se halló que los participantes con menor edad presentaban puntuaciones más elevadas en la dimensión de ahorro $(r=$ $-.27 ; p<0.001)$, mientras que altas puntuaciones en manejo de la deuda $(r=.43 ; p<0.001)$ o del dinero $(r=.22 ; p<0.001)$ se asociaban con una mayor edad del participante.

Con respecto a las comparaciones por sexo, solo se hallaron diferencias significativas en cuanto a las conductas de ahorro $(t=-5.44 ; g l=$ $778 ; p<0.001$ ), siendo los hombres los que pre- 
Tabla 3

Evidencia de validez externa del test.

\begin{tabular}{llllll}
\hline Correlaciones & MDE & MDI & IC & PC & URC \\
\hline Ahorro (A) & $.16^{* *}$ & .05 & $.21^{* *}$ & $.16^{* *}$ & $.09^{*}$ \\
Manejo de la Deuda (MDE) & 1 & $.13^{* *}$ & .04 & $.20^{* *}$ & $.45^{* *}$ \\
Manejo del Dinero (MDI) & & 1 & $-.23^{* *}$ & $.46^{* *}$ & $.31^{*}$ \\
Impulsividad de la Compra (IC) & & 1 & $.11^{* *}$ & .05 \\
Planificación de la Compra (PC) & & & 1 & $.51^{* *}$ \\
\hline
\end{tabular}

Nota. URC $=$ uso responsable del crédito y calidad del producto. Esta dimensión combina dos factores como resultado del análisis factorial de la presente investigación.

** La correlación de producto-momento de Pearson es significativa en el nivel .01 (2 colas).

sentaron mayores comportamientos ligados a la preservación de su patrimonio $(\mathrm{M}=3.00$; $\mathrm{DE}=$ .73) en comparación con las mujeres $(\mathrm{M}=2.72$; $\mathrm{DE}=.63)$. No obstante, el tamaño del efecto de esa diferencia fue baja $(d$ de Cohen $=.29)$.

Por último, todas las dimensiones de la conducta financiera presentaron diferencias en función de la ocupación del participante. Concretamente, para las conductas de ahorro $(\mathrm{F}=$ 31.37; $g l=779$; $p<0.001)$, los patronos y trabajadores por cuenta propia tienden a presentar una puntuación significativamente mayor $(\mathrm{M}=3.09)$ a la de los trabajadores privados o públicos $(\mathrm{M}=$ 2.82; $\mathrm{M}=2.30$ ), que son los grupos que manifestaron menor propensión al ahorro.

Por otro lado, para el caso del manejo de la deuda $(\mathrm{F}=28.32 ; g l=779 ; p<0.001)$, los patronos y trabajadores por cuenta propia puntúan significativamente más alto en el acceso que tienen a dicha oportunidad si se les compara con los estudiantes de universidades privadas y públicas o con los trabajadores del sector privado. Resultados similares se encuentran en el manejo del dinero $(\mathrm{F}=2.81 ; g l=779 ; p=.025)$, ya que los patrones tienden a diferenciarse significativa- mente si se les contrasta con el resto.

Para finalizar, tomando en consideración que el $54.6 \%$ de los sujetos no poseía tarjeta de crédito al momento del estudio producto de las políticas de encaje legal en el país, se procedió a extraer de la escala los ítems que hacían referencia explícita al uso de tarjetas de crédito (ítems 2 , 11 y 23).

Lo anterior se hizo con la finalidad de verificar por medio de tres análisis factoriales exploratorios si el comportamiento de las cargas de la escala variaba en monto y factor al comparar tres grupos: personas que tenían tarjetas de crédito $(\mathrm{n}=348)$, personas que no tenían $(\mathrm{n}=432)$ y la muestra completa $(n=780)$. Se encontró que las cargas de los ítems se mantenían en los mismos factores, pero aumentaban ligeramente. Esto indica que al utilizar estos tres ítems se debe garantizar que la muestra sea homogénea en cuanto al acceso a tarjetas de crédito.

\section{Discusión}

Dado que los contextos de adversidad tien- 
den a modificar las conductas financieras de los individuos y que además no se encontraron en la literatura propuestas operacionales que permitieran obtener indicadores contextualizados del comportamiento económico de las personas en situaciones problemáticas, se propuso la construcción de una escala que cumpliera con dichos fines. En primer lugar, se garantizó el proceso de validación de contenido del instrumento mediante las siguientes fases: (a) la revisión de la literatura para la familiarización con la conducta financiera como constructo, (b) la elaboración de una matriz de especificaciones que contemplara el contenido de los ítems y su formato de respuesta, (c) la construcción de un banco de ítems que contuvo el doble de afirmaciones estipuladas, (d) la evaluación del banco de ítems por parte de tres jueces expertos que emitieron una valoración en términos de pertinencia, claridad y adecuación de los ítems a la muestra; y (e) la elección de una versión preliminar de la escala que fue aplicada a una muestra piloto con el objetivo de obtener las propiedades psicométricas de las puntuaciones del test (Muñiz \& Fonseca-Pedrero, 2019; Pérez \& Olaz, 2008).

Con respecto al segundo objetivo de la investigación, se encontraron tres factores que fueron denominados (a) ahorro, (b) manejo de la deuda y (c) manejo del dinero. El primer factor se refiere a la abstención en la utilización del dinero en un determinado momento con la finalidad de gastarlo en un futuro. El segundo factor implica el préstamo de dinero por parte de una entidad financiera (o proveedores informales); evalúa cómo actúan los sujetos ante estas deudas. Por último, el resto de afirmaciones presentó un conjunto de acciones que denotaron la planificación y organización del uso que las personas le dan a su dinero en transacciones y pagos cotidianos (Dew \& Xiao, 2011; Van Raaij, 2016).

Esta estructura factorial obtenida a partir de la muestra del presente estudio mantuvo una semejanza con las propuestas operacionales de Dew y Xiao (2011) y Ksendzova et al. (2017). En paralelo, resultó ser una agrupación de dimensiones cercanas a explicar el $49.69 \%$ de la varianza total del test, condición sugerida en la interpretación de análisis factorial exploratorio (Pérez \& Olaz, 2008).

En cuanto a la consistencia interna del instrumento, las puntuaciones de las dimensiones fueron adecuadas para fines de investigación siguiendo las sugerencias de la literatura (Prieto \& Muñiz, 2008) en el caso del ahorro y el manejo de la deuda. Este planteamiento se asemeja a los hallazgos de la escala de Ksendzova et al. (2017) y se diferenció del instrumento original de Dew y Xiao (2011), el cual presentó inconvenientes en la fiabilidad de la dimensión referida al manejo del crédito.

No obstante, el presente instrumento halló para las puntuaciones de manejo del dinero una consistencia interna que según los parámetros de Prieto y Muñiz (2008) presenta ciertas fallas, siendo entonces una medida susceptible de ser mejorada. Resultados similares fueron informados para dicho factor en la escala de Dew y Xiao (2011), ya que, según los autores, esta propiedad de la conducta financiera tiende a poseer ítems de difícil redacción.

Tomando en consideración la estructura factorial del test y su fiabilidad, se eliminaron tres afirmaciones en la escala (ítems 6: Ahorro gran porcentaje del dinero que recibo en bolivares, 8: Pago atrasado a mis amigos o allegados cuando me toca devolver el dinero, y 15: Utilizo una proporción de mis ingresos en bolivares para comprar bienes duraderos), que según el estudio realizado sobre la base del análisis factorial exploratorio no formaban parte de ninguna dimensión.

La ausencia de carga de estos ítems sugería su eliminación. En primer lugar, en el caso del 
sexto ítem, considerando el contexto económico venezolano (España \& Ponce, 2018), aquellas personas que deciden ahorrar en bolívares y no en divisas extranjeras son sujetos con poca capacidad de entendimiento de las dinámicas económicas en contextos de adversidad, y, por tanto, esto no se asocia con ningún comportamiento financiero asertivo.

Este bajo entendimiento de las dinámicas inflacionarias también podría explicar la ausencia de carga en el octavo ítem. En estos contextos, percibir una deuda en la moneda a devaluarse y pagarla de forma retrasada en el tiempo, sería una estrategia para que el monto a cancelar sea mucho menor al adquirido en la deuda inicial.

Sin embargo, alrededor de un $80 \%$ de la muestra indicó que Nunca o Casi nunca adopta dicha estrategia, elemento que sugiere que la negativa a dicha posibilidad es propia de sujetos con un peor uso del dinero en un país hiperinflacionario. O bien, también podría simplemente sugerir que los sujetos de la muestra encuestada prefieren no endeudarse ante la realidad actual, no presentando estas conductas; aspecto que es muy similar al del ítem 15.

Con respecto al último objetivo, las puntuaciones de la escala presentaron evidencia de validez convergente en la medida en que todas las dimensiones de la conducta financiera se asociaron positiva y significativamente con otras medidas preexistentes de planificación de la compra, uso responsable del crédito y de la calidad de servicio (Denegri et al., citado en Ortega \& RodríguezVargas, 2004), tres de los indicadores subyacentes en la medición adecuada de la dimensión de ahorro, manejo de la deuda y del dinero (Pérez \& Olaz, 2008).

En el caso de la validez discriminante, las dimensiones de manejo de la deuda y del dinero obtuvieron un patrón relacional negativo o nulo respecto a la medida impulsividad de la compra, resultado apropiado desde un punto de vista psicométrico (Perez \& Olaz, 2008) en la medida en que se demuestra que estos factores de la conducta financiera se diferencian de aquellos constructos que se suponen teóricamente opuestos.

No obstante, para el caso de la dimensión del ahorro, se obtuvo una correlación inesperada entre esta y la impulsividad de la compra, ya que la asociación resultó baja y positiva. Es posible que esta relación se sustente en cómo las personas buscan sobrellevar el ritmo inflacionario de la moneda local: debido a que el bolívar no se constituye como una moneda estable, una alternativa de ahorro en Venezuela se presenta en la compra impulsiva de divisas o bienes duraderos.

En cuanto a la asociación entre las dimensiones de la conducta financiera, se halló un predominio de relaciones bajas. Específicamente, la asociación entre el ahorro y el manejo de la deuda, y entre este último y el manejo del dinero, aunque significativas, resultaron bajas. Esto se debe a que las personas con recursos económicos abundantes no tienden a preocuparse por la planificación que hacen del uso del dinero en términos de créditos y de manejo del efectivo. No es este el caso de las personas con dificultades económicas, quienes tienden a no endeudarse y a planificar con rigurosidad el uso de sus recursos financieros.

Por otra parte, se encontró que la escala presentó un funcionamiento diferencial de sus dimensiones en función del sexo de los sujetos, la ocupación actual y la edad. Estos hallazgos pueden considerarse como un elemento que permita en posteriores investigaciones obtener indicadores de validez de criterio que no fueron desarrollados en este estudio.

Por último, en cuanto a las limitaciones del estudio, se encontró el tipo de muestreo no probabilístico, la exclusión de participantes con otras ocupaciones laborales (p. ej., cargos obreros), y el hecho de que para utilizar esta escala en una 
muestra ajena a la población venezolana se debe realizar una adaptación leve de los ítems, de forma que excluyan elementos propios de la dinámica económica del país (p. ej., la sustitución de la moneda local). Por otra parte, para poder utilizar la escala con los tres ítems que hacen referencia al uso de tarjetas de crédito, se debe garantizar que la mayoría de los sujetos encuestados tengan acceso a este tipo de beneficio financiero.

\section{Referencias}

Barbar, R., Benasayag, S., \& Salomón, L. (2019, enero). Nuevo encaje bancario: ¿Cuáles son las implicaciones? PRODAVINCI. Recuperado de https://prodavinci.com

Ranyard, R., \& De Mello-Ferreira, V. R. (2017). Introduction to economic psychology: The science of economic mental life and behaviour. En R. Ranyard (Ed.), Economic Psychology (pp. 1-18). doi: 10.1002/9781118926352.ch1

De Minzi, M. (2008). Nuevas tendencias en psicometría. Revista Evaluar, 8(1), 1-19. doi: 10.35670/16674545.v8.n1.501

Dew, J., \& Xiao, J. J. (2011). The financial management behavior scale: Development and validation. Journal of Financial Counseling and Planning, 22(1), 43-59. Recuperado de https://digitalcommons.uri.edu

España, L., \& Ponce, M. (2018). Evolución de la pobreza. IIES/UCAB. Recuperado de https://www.ucab.edu. ve

Fernández, A., Pérez, E., Alderete, A. M., Richaud, M. C., \& Liporace, M. F. (2010). ¿Construir o adaptar tests psicológicos? Diferentes respuestas a una cuestión controvertida. Revista Evaluar, 10(1), 60-74. doi: 10.35670/1667-4545.v10.n1.459

Garðarsdóttir, R. B., \& Dittmar, H. (2012). The relationship of materialism to debt and financial well-being: The case of Iceland's perceived prosperity. Journal of Economic Psychology, 33(3), 471-481. doi:

\subsection{6/j.joep.2011.12.00}

Gärling, T., Kirchler, E., Lewis, A., \& Van Raaij, F. (2009). Psychology, financial decision making, and financial crises. Psychological Science in the Public Interest, 10(1), 1-47. doi: 10.1177/1529100610378438

Gudmunson, C. G., \& Danes, S. M. (2011). Family financial socialization: Theory and critical review. Journal of Family and Economic Issues, 32(4), 644-667. doi: 10.1007/s10834-011-9275-y

Gudmunson, C., Ray, S. K., \& Xiao, J. J. (2016). Financial socialization. En J. J. Xiao (Ed.), Handbook of Consumer Finance Research (pp. 61-72). Cham: Springer. doi: 10.1007/978-3-319-28887-1_5

Hair, J. F., Black, W. C., Babin, B. J., \& Anderson, R. E. (2019). Multivariate Data Analysis. Estados Unidos: Cengage.

IBM Corp. (2013). IBM. SPSS Statistics for Windows (Version 22.0). [Software de cómputo]. Armonk, NY: IBM Corp.

Kahneman, D. (2013). Pensar rápido, pensar despacio. Estados Unidos: Debolsillo.

Kerlinger, F., \& Lee, H. (2002). Investigación del comportamiento: Métodos de Investigación en Ciencias Sociales. ( $4^{\text {a }}$ ed.). Ciudad de México, México: McGraw Hill.

Kirchler, E., \& Hoelzle, E. (2018). Economic Psychology: An Introduction. Cambridge University Press: United Kingdom.

Ksendzova, M., Donnelly, G. E., \& Howell, R. T. (2017). A brief money management scale and its associations with personality, financial health, and hypothetical debt repayment. Journal of Financial Counseling and Planning, 28(1), 62-75. doi: 10.1891/1052-3073.28.1.62

Laibson, D., \& List, J. A. (2015). Principles of (behavioral) economics. American Economic Review, 105(5), 385-390. doi: 10.1257/aer.p20151047

Lloret-Segura, S., Ferreres-Traver, A., Hernández-Baeza, A., \& Tomás-Marco, I. (2014). El análisis factorial exploratorio de los ítems: Una guía práctica, revisada y actualizada. Anales de Psicología, 30(3), 1151- 
1169. doi: 10.6018/analesps.30.3.199361

Lorenzo-Seva, U., \& Ferrando, P. (2011). FACTOR (Versión 8.02). [Software de cómputo]. Recuperado de http://psico.fcep.urv.es/utilitats/factor/index.html

Magnusson, D. (1972). Teoría de los test. Ciudad de México, México: Trillas.

Manzini, J. L. (2000). Declaración de Helsinki: Principios éticos para la investigación médica sobre sujetos humanos. Acta Bioethica, 6(2). doi:10.4067/ S1726-569X2000000200010

Mcnair, S., \& Crozier, W. R. (2017). Assessing psychological dispositions and states that can influence economic behaviour. En R. Ranyard (Ed.), Economic Psychology (pp. 69-87). Hoboken: Wiley-Blackwell. doi: 10.1002/9781118926352.ch5

Montero, I., \& León, O. G. (2007). A guide for naming research studies in Psychology. International Journal of Clinical and Health Psychology, 7(3), 847-862. Recuperado de https://www.redalyc.org

Muñiz, J., \& Fonseca-Pedrero, E. (2019). Diez pasos para la construcción de un test. Psicothema, 31(1), 7-16. doi: 10.7334/psicothema2018.291

Olmo, D. G. (18 de noviembre de 2019). Dolarización en Venezuela: Cómo Nicolás Maduro cambió de opinión sobre el dólar y su papel en la economía. $B B C$ News. Recuperado de https://www.bbc.com/mundo/ noticias-america-latina-50466818

Ortega, V., \& Vargas, J. C. R. (2004). Escala de hábitos y conductas de consumo: Evidencias sobre dimensionalidad. International Journal of Clinical and Health Psychology, 4(1), 121-136. Recuperado de https:// www.redalyc.org

Pérez, E., \& Olaz, F. (2008). Validez. En S. Tornimbeni, E. Pérez \& F. Olaz (Eds.), Introducción a la Psicometría (pp.101-136). Buenos Aires, Argentina: Paidós.

Perry, V. G., \& Morris, M. D. (2005). Who is in control? The role of self-perception, knowledge, and income in explaining consumer financial behavior. Journal of Consumer Affairs, 39(2), 299-313. doi: 10.1111/j.1745-6606.2005.00016.x

Porter, N. M., \& Garman, E. T. (1993). Testing a conceptual model of financial well-being. Financial Counseling and Planning, 4, 135-165. doi: 10.1.1.379.6832

Prieto, G., \& Muñiz, J. (2000). Un modelo para evaluar la calidad de los test utilizados en España. Papeles del Psicólogo, 77, 65-75. Recuperado de https://www. redalyc.org

Sardi, G., Angelucci, L., Martins, A., \& Peña, G. Representaciones de la economía como fenómeno psicosocial. En C. Peña (Ed.). Miradas a la Venezuela del siglo XXI (pp. 377-406). Caracas, Venezuela: Instituto de Investigaciones Económicas y Sociales: "Dr. Rodolfo Quintero".

Veiga, R. T., Avelar, C., Moura, L. R. C., \& Higuchi, A. K. (2019). Validation of scales to research the personal financial management. Revista Brasilera de Gestão de Negócios, São Paulo, 21(2), 332-348. doi: 10.7819/rbgn.v21i2.3976

UCAB. (2020, 13 de julio). UCAB ENCOVI | 2019-2020. Recuperado de https://www.youtube.com/watch?reload $=9 \& \mathrm{v}=$ ph0QyIaWJ_k\&feature=youtu.be

Van Raaij, W. F. (1981). Economic psychology. Journal of Economic Psychology, 1(1), 1-24. doi: 10.1016/0167-4870(81)90002-7

Van Raaij, W. F. (2016). Understanding consumer financial behavior money management in an age of financial illiteracy. doi: 10.1057/9781137544254

VandenBos, G. R. (2015). APA Dictionary of Psychology ( $2^{\text {a }}$ ed.). USA: American Psychological Association. doi: 10.1037/14646-000

Xiao, J. J. (2008). Appliying behavior theories to financial behavior. En J. J. Xiao (Ed.), Handbook of Consumer Finance Research (pp. 69-81). New York, USA: Springer. doi: 10.1007/978-0-387-75734-6_5 